\title{
Precision measurement of the proton and helium flux in primary cosmic rays with the Alpha Magnetic Spectrometer on the Inter- national Space Station
}

\author{
M. Heil ${ }^{1, a}$ On behalf of the AMS collaboration \\ ${ }^{1}$ Massachusetts Institute of Technology (MIT), Cambridge, Massachusetts 02139, USA
}

\begin{abstract}
The precise measurements of the proton and helium flux in primary cosmic rays based on on data collected by the Alpha Magnetic Spectrometer during the first 30 months of operation (May 19, 2012 to November 26, 2013) onboard the International Space Station are presented. Knowledge of the rigidity dependence of the proton and helium flux is important in understanding the origin, acceleration, and propagation of cosmic rays in our galaxy. The high statistics of the measurements (300 mio. protons, 50 mio. helium) allow to study the detailed variations with rigidity of the fluxes spectral index. The spectral index of both the proton and the helium flux progressively hardens at rigidities larger than $100 \mathrm{GV}$. The rigidity dependence of the helium flux spectral index is similar to that of the proton spectral index though the magnitudes are different. Remarkably, the spectral index of the proton to helium flux ratio increases with rigidity up to 45 $\mathrm{GV}$ and then becomes constant; the flux ratio above $45 \mathrm{GV}$ is well described by a single power law.
\end{abstract}

\section{Introduction}

Protons and helium nuclei in cosmic rays are believed to be mainly produced by Galactic sources such as supernova remnants. The precise proton and helium spectra in the GV - TV rigidity region give important information on the origin, acceleration, and subsequent propagation processes of cosmic rays in the Galaxy [1].

Some of the recent important measurements of the proton flux in cosmic rays are listed in Ref. [26]. Recent important measurements of the helium flux in cosmic rays have reported different variations of the flux with energy [4, 6-9]. Many models based on different sources, acceleration mechanisms, and diffusive propagation effects [10] were proposed to account for the hardening of the helium flux as well as for the difference of the proton and helium fluxes. The proton flux was reported in Ref. [11] and the helium flux was reported in Ref. [12] both based on data collected by the Alpha Magnetic Spectrometer (AMS) during the first 30 months (May 19, 2011 to November 26, 2013) of operation onboard the International Space Station (ISS). The Letter reporting the helium flux also reports the characteristics of the ratio of the proton flux to the helium flux.

ae-mail: melanie.heil@ cern.ch 


\section{Detector}

AMS is a general purpose high energy particle physics detector in space. The layout and description of the detector are presented in Ref. [13]. The key elements used in this measurement are the permanent magnet, the silicon tracker, four planes of time of flight (TOF) scintillation counters, and the array of anticoincidence counters (ACC). AMS also contains a transition radiation detector (TRD), a ring imaging Čerenkov detector (RICH), and an electromagnetic calorimeter (ECAL). The central field of the magnet [14] is $1.4 \mathrm{kG}$.

The tracker [15] has nine layers, the first (L1) at the top of the detector, the second (L2) just above the magnet, six (L3 to L8) within the bore of the magnet, and the last (L9) just above the ECAL. L2 to L8 constitute the inner tracker. Each layer contains double-sided silicon microstrip detectors which independently measure the $x$ and $y$ coordinates. The tracker accurately determines the trajectory of cosmic rays by multiple measurements of the coordinates with a resolution in each layer of 7-10 $\mu \mathrm{m}$ in the bending $(y)$ direction. The inner tracker is held stable by a carbon fiber structure with negligible coefficient of thermal expansion. The stability of the inner tracker is monitored using 20 IR laser beams which penetrate layers L2 through L8 and provide sub-micron position measurements. Using cosmic rays over a 2 minute window, the position of $\mathrm{L} 1$ is aligned with a precision of $5 \mu \mathrm{m}$ with respect to the inner tracker and L9 with a precision of $6 \mu \mathrm{m}$. Together, the tracker and the magnet measure the rigidity $R$ of charged cosmic rays. The maximum detectable rigidity (MDR) for particles with charge $|Z|=1$ is $2 \mathrm{TV}$ over the $3 \mathrm{~m}$ lever arm from L1 to L9. For particles with charge $|Z|=2$ the MDR is higher, namely 3.2 TV, as the higher signal amplitudes of $|Z|=2$ particles provide a better coordinate resolution.

Each layer of the tracker also provides an independent measurement of the absolute value of the charge $|Z|$ of the cosmic ray. The charge resolution of the layers of the inner tracker together is $\Delta Z \simeq 0.05$ for $|Z|=1$ particles and $\Delta Z \simeq 0.07$ for $|Z|=2$.

Two planes of TOF counters [16] are located above L2 and two planes are located below the magnet. The overall velocity $(\beta=v / c)$ resolution has been measured to be $\Delta \beta / \beta^{2}=4 \%$ for $|Z|=1$ particles and $\Delta \beta / \beta^{2}=2 \%$ for $|Z|=2$ particles. This discriminates between upward- and downwardgoing particles. The pulse heights of the two upper layers and of the two lower layers are combined to provide two independent measurements of the charge.

The 16 anticoincidence counters (ACC) form a cylindrical shell between the inner tracker and the magnet. Adjacent counters are combined to provide 8 readout sectors. The ACC have an efficiency of 0.99999 to reject cosmic rays which enter the inner tracker from the side.

Before launch, at the CERN SPS, AMS was extensively calibrated with 180 and $400 \mathrm{GeV} / c$ proton beams and beams of positrons, electrons, and pions from 10 to $290 \mathrm{GeV} / c$. In total, calibrations with 18 different energies and particles at 2000 positions were performed. These data allow the determination of the tracker rigidity resolution function with high precision and the verification of the absolute rigidity scale.

Monte Carlo simulated events were produced using a dedicated program developed by the collaboration based on the GEANT-4.9.6 package for protons and the GEANT-4.10.1 package for helium [17]. This program simulates electromagnetic and hadronic interactions of particles in the material of AMS and generates detector responses. The INCL++ package [18] was used to model helium-nuclear inelastic interactions below $5 \mathrm{GeV} /$ nucleon and the DPMJET-II.5 package [19] was used at higher energies. The helium-nuclear elastic and quasi-elastic scattering were modeled using the measurements from [20]. As will be discussed below, we have developed a method to measure the helium-nuclear interactions with the materials in AMS and used this measurement to scale the model predictions.

The digitization of the signals is simulated precisely according to the measured characteristics of the electronics. The simulated events then undergo the same reconstruction as used for the data. 
Figure 1(a) shows a comparison of the inverse rigidity for $400 \mathrm{GeV} / c$ protons from the test beam and the Monte Carlo simulation. As seen, the resolution has a pronounced Gaussian core with nonGaussian tails of $\sim 5 \%$ and the simulation precisely reproduces the measured resolution including the tails.

\section{Selection}

In the first 30 months $\left(7.96 \times 10^{7} \mathrm{~s}\right)$ AMS collected $4.1 \times 10^{10}$ cosmic ray events. The effective data collection time used in this analysis are the seconds when the detector was in normal operating conditions, the AMS $z$ axis was within $40^{\circ}$ of the local zenith, the trigger live time exceeded $50 \%$, and the ISS was outside of the South Atlantic Anomaly. Due to the influence of the geomagnetic field, this collection time for primary cosmic rays increases with increasing rigidity becoming constant at $6.29 \times 10^{7}$ s above $30 \mathrm{GV}$.

By selecting events to be downward going and to have a reconstructed track in the inner tracker with $|Z|=1$, we obtain $1.1 \times 10^{10}$ proton candidate events. By selecting $|Z|=2$ in the inner tracker we obtain $1.7 \times 10^{9}$ helium candidate events. In order to have the best resolution at the highest rigidities, further selections are made by requiring the track to have hits in L1 and L9 with reconstructed charges compatible to that of the inner tracker and to satisfy additional track fitting quality criteria such as a $\chi^{2} /$ d.f. $<10$ in the bending coordinate. To further remove helium candidates which interacted within the detector, the charge measured by the upper TOF and the lower TOF is also required to be compatible with $Z=2$,

In addition, to select only primary cosmic rays, well above the geomagnetic cutoff, the measured rigidity is required to be greater than 1.2 times the maximum geomagnetic cutoff within the AMS field of view. The cutoff was calculated by backtracing [21] particles from the top of AMS out to 50 Earth's radii using the IGRF [22] geomagnetic model. For protons the small contamination of low energy (below $2 \mathrm{GeV}$ ) pions produced in the upper part of the detector is removed by requiring that the mass of the selected particle is larger than $0.5 \mathrm{GeV} / c^{2}$. These procedures resulted in a sample of $3.0 \times 10^{8}$ primary cosmic rays with $Z=+1$ and $5.0 \times 10^{7}$ primary cosmic rays with $Z=+2$.

Since protons are the dominant component of cosmic rays, the selected sample of $3.0 \times 10^{8}$ events has only small contributions of other particles, mainly deuterons. The deuteron contribution decreases with rigidity; at $1 \mathrm{GV}$ it is less than $2 \%$ and at $20 \mathrm{GV}$ it is $0.6 \%[23,24]$. Deuterons were not removed. The sample also contains protons from nuclei which interact at the top of AMS (for example, in L1 or the TRD). From the measured flux $[12,25]$ and Monte Carlo simulation this contribution is $0.5 \%$ at $1 \mathrm{GV}$ decreasing to less than $0.1 \%$ at and above $10 \mathrm{GV}$. Contamination from $e^{+}$and $e^{-}$[26] was estimated to be less than $0.1 \%$ over the entire rigidity range. Due to the multiple independent measurements of the charge, the selected helium sample contains only a small contamination of particles which had $Z \neq 2$ at the top of AMS. Comparing the proton and helium charge distributions in the inner tracker, the proton contamination of the helium sample was measured to be less than $10^{-4}$ over the entire rigidity range. The sample also contains helium from other nuclei which interact at the top of AMS (for example, in L1). From the measured flux [25] and Monte Carlo simulation this contribution is below $10^{-3}$ for the entire rigidity range. The background contributions for protons and helium are subtracted from the fluxes and the uncertainties are accounted for in the systematic errors.

\section{Analysis}

The isotropic flux $\Phi_{i}$ for the $i^{\text {th }}$ rigidity bin $\left(R_{i}, R_{i}+\Delta R_{i}\right)$ is

$$
\Phi_{i}=\frac{N_{i}}{A_{i} \epsilon_{i} T_{i} \Delta R_{i}}
$$


where $N_{i}$ is the number of events corrected with the rigidity resolution function (see below), $A_{i}$ is the effective acceptance, $\epsilon_{i}$ is the trigger efficiency, and $T_{i}$ is the collection time. The effective acceptance $A_{i}$ was calculated using Monte Carlo samples and then corrected for small differences found between the data and Monte Carlo event selection efficiencies.

The trigger efficiency $\epsilon_{i}$ is measured from data with the unbiased trigger events $[11,12]$. The trigger efficiency of protons ranges from 90 to $95 \%$, while the trigger efficiency for helium was measured to range from 95 to $99.5 \%$ The inefficiencies are due to secondary $\delta$-rays in the magnetic field entering the ACC. The Monte Carlo trigger efficiencies agree with the measured trigger efficiencies within $0.5 \%$.

The bin-to-bin migration of events was corrected using the rigidity resolution functions obtained from Monte Carlo simulations and for protons verified with the test beam data, see for example Fig. 1(a).
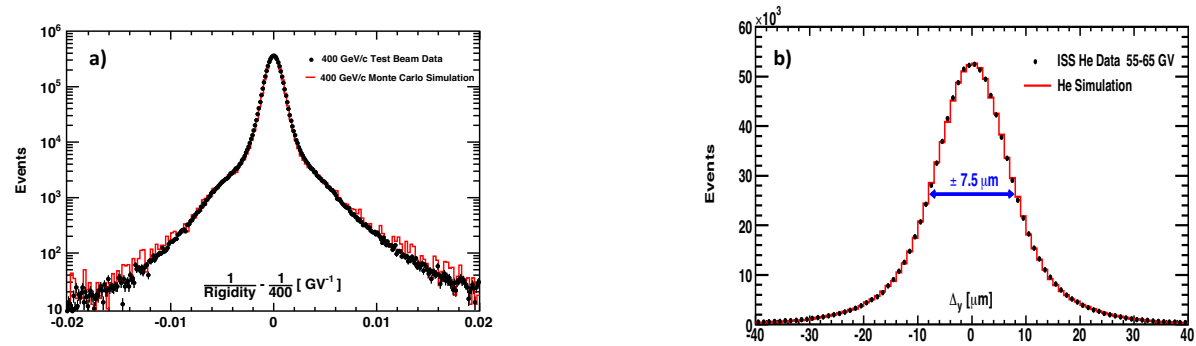

Figure 1: a) The resolution function in inverse rigidity for $400 \mathrm{GeV} / c$ protons measured in the test beam compared with Monte Carlo simulated events. b) The differences of the coordinates measured in the tracker layers 3 or 5 to those obtained from the track fit excluding the tested layer for helium events for both data and Monte Carlo simulation.

Among many unfolding procedures, we selected two which are described in [11]. The validity of both were verified by our Monte Carlo simulation. The small differences between the two procedures $(<0.5 \%)$ are accounted as a systematic error. We have checked the sensitivity of the results to the binning by increasing the bin width by factors of 2 and 4 as well as reducing the bin width by factors of 2 and 4 . The resulting uncertainty is well within the assigned systematic errors.

Extensive studies were made of the systematic errors. The errors include the uncertainties in the trigger efficiency, the acceptance, the background contamination also accounting for helium interactions in the detector, the geomagnetic cutoff factor, the event selection, the unfolding, the rigidity resolution function, and the absolute rigidity scale. The trigger efficiency error is dominated by the statistics available from the $1 \%$ prescaled unbiased event sample $[11,12]$. For protons it is negligible (less than $0.1 \%$ ) below $500 \mathrm{GV}$ and reaches $1.5 \%$ at $1.8 \mathrm{TV}$, for helium it is less than $0.2 \%$ below $100 \mathrm{GV}$ and reaches $1 \%$ at $3 \mathrm{TV}$. The geomagnetic cutoff factor was varied from 1.0 to 1.4 and the resulting fluxes showed a systematic uncertainty of $2 \%$ at $1 \mathrm{GV}$ and negligible above $2 \mathrm{GV}$.

The effective acceptance was corrected for small differences between the data and the Monte Carlo samples related to the event reconstruction and selection. The total correction for protons was found to be $5 \%$ at $1 \mathrm{GV}$ decreasing below $2 \%$ above $10 \mathrm{GV}$, while the corresponding systematic uncertainty is less than $1 \%$ above $2 \mathrm{GV}$. The correction on the acceptance for helium was found to be less than $2 \%$ above $2 \mathrm{GV}$ with a corresponding systematic error on the flux less than $1 \%$ below $200 \mathrm{GV}$ and reaching $1.4 \%$ at $3 \mathrm{TV}$. 
The detector is mostly made of carbon and aluminum. For protons the corresponding inelastic cross sections of $p+\mathrm{C}$ and $p+\mathrm{Al}$ are known to within $10 \%$ at $1 \mathrm{GV}$ and $4 \%$ at $300 \mathrm{GV}$ [27], and $7 \%$ at $1.8 \mathrm{TV}$ from model estimations [17]. To estimate the systematic error of the proton flux due to the uncertainty in the inelastic cross sections, dedicated samples of protons were simulated with the $p+\mathrm{C}$ and $p+\mathrm{Al}$ cross sections varied by $\pm 10 \%$. From the analysis of these samples together with the current knowledge of the cross sections, a systematic error of $1 \%$ at $1 \mathrm{GV}, 0.6 \%$ from 10 to $300 \mathrm{GV}$, and $0.8 \%$ at $1.8 \mathrm{TV}$ was obtained. The inelastic cross sections of $\mathrm{He}+\mathrm{C}$ and $\mathrm{He}+\mathrm{Al}$ have only been measured below $10 \mathrm{GV}$ [28]. To accurately determine the effect on the acceptance of helium interactions in the detector, we have developed a method [29] to determine the magnitude and rigidity dependence of the survival probability of helium nuclei when traversing the detector materials. For this we used a sample of primary cosmic rays collected with AMS horizontal, that is, when the ISS was oriented such that AMS was pointing within $90^{\circ} \pm 10^{\circ}$ of the local zenith, a total of $1.4 \times 10^{5} \mathrm{~s}$. In this condition, primary helium can travel from L9 to L1 and from L1 to L9. For more details see [12]. Using the measured interaction probabilities and, in addition, above $100 \mathrm{GV}$ the rigidity dependence of the cross sections from the Glauber-Gribov model [17], the systematic error on the flux due to uncertainties of helium inelastic cross sections was evaluated to be $1 \%$ below $100 \mathrm{GV}$ and $2 \%$ at $3 \mathrm{TV}$.

An additional systematic error on the helium flux is due to differences in ${ }^{3} \mathrm{He}$ and ${ }^{4} \mathrm{He}$ interactions with the detector materials. This error was assigned according to the uncertainty in the helium isotopic composition. The error of $0.3 \%$ on the flux was obtained by varying the ratio of ${ }^{3} \mathrm{He} /{ }^{4} \mathrm{He}$ in the simulation from 0 to 0.2 [30]. The helium flux was then treated as containing only ${ }^{4} \mathrm{He}$.

The rigidity resolution function for protons, shown in Fig. 1(a), was verified with data from both the ISS and the test beam and compared to the simulated events. For this the residuals between the hit coordinates measured in tracker layers L1 and L9 and those obtained from the track fit using the information from only the inner tracker L2 to L8 were compared between data and simulation. In order to validate the alignment of the external layers the difference between the rigidity measured using the information from L1 to L8 and from L2 to L9 was compared between data and the simulation. The resulting uncertainty on the proton MDR was estimated to be 5\%. The corresponding unfolding errors on the proton flux were obtained by varying the width of the Gaussian core of the resolution function by $5 \%$ and the amplitude of the non-Gaussian tails by $\sim 20 \%$ over the entire rigidity range and found to be $0.8 \%$ below $200 \mathrm{GV}$ and $3 \%$ at $1.8 \mathrm{TV}$.

The rigidity resolution function for helium is similar to that of the protons. It was obtained from the simulations and extensively verified with the data. We list three verifications. First, the differences of the coordinates measured in the inner tracker layers to those obtained from the track fit excluding the tested layer were compared between data and simulation. This procedure directly measures the tracker coordinate accuracy of $\pm 7.5 \mu \mathrm{m}$ for helium as shown in Fig. 1(b) for the combination of Layers 3 and 5. Second, the differences between the coordinates measured in L1 and L9 and those obtained from the track fit using the information from only the inner tracker were compared between helium events in data and simulation. Third, the RICH velocity resolution is $\Delta \beta / \beta=8.0 \times 10^{-4}$ [31] for helium events. The rigidity resolution function up to $20 \mathrm{GV}$, including non-Gaussian tails, was obtained with data using the RICH velocity measurements only and compared with the rigidity resolution function from the simulation. The first two verifications provided the helium MDR and its uncertainty of $3.2 \pm 0.16 \mathrm{TV}$. The second and third provided the uncertainty of the amplitude of the non-Gaussian tails in the helium rigidity resolution function to be $10 \%$. The systematic error on the helium flux due to uncertainties in the rigidity resolution function was obtained by varying the width of the Gaussian core of the resolution function by $5 \%$ and the amplitude of the non-Gaussian tails by $10 \%$ over the entire rigidity range in the unfolding procedures and found to be less than $1 \%$ below $400 \mathrm{GV}$ and $3.5 \%$ at $3 \mathrm{TV}$. 
There are two contributions to the systematic uncertainty on the rigidity scale. The first is due to residual tracker misalignment. From the $400 \mathrm{GeV} / c$ proton test beam data it was measured to be less then $1 / 300 \mathrm{TV}^{-1}$. For the ISS data this error was estimated by comparing the $E / p$ ratio for electron and positron events, where $E$ is the energy measured with the ECAL and $p$ is the momentum measured with the tracker, see Ref. [32] for details. It was found to be $1 / 26 \mathrm{TV}^{-1}$, limited by the current high energy positron statistics. The second systematic error on the rigidity scale arises from the magnetic field map measurement and its temperature corrections and amounts to less than $0.5 \%$ for rigidities above $2 \mathrm{GV}$.

To ensure that the treatment of systematic errors described above is correct, we performed several additional, independent verifications. Figure 2 shows examples of the stability of the measured proton flux for different conditions (presented as the ratio to the average flux) on the left and examples of the stability of the measured helium flux for different conditions (presented as the ratio to the average flux) along with the corresponding systematic errors on the right.
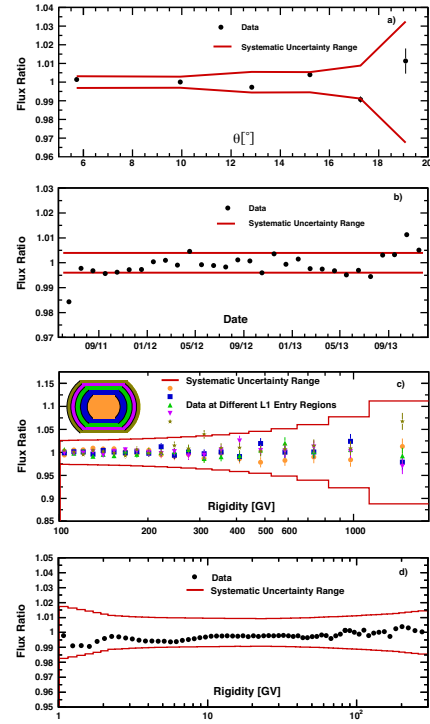
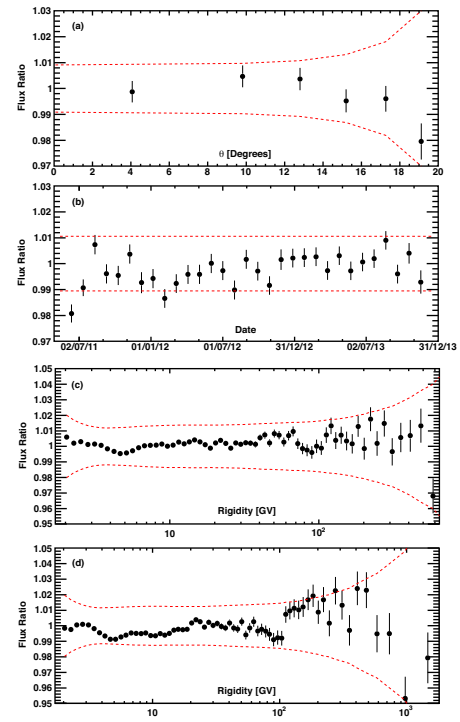

Figure 2: Verifications of the systematic errors for the proton (left) and helium (right) flux measurement. a) The variation of the flux ratio above $30 \mathrm{GV}$ versus the angle $\theta$ to the AMS $z$ axis. b) The variation of the flux ratio above $45 \mathrm{GV}$ vs time. c) (left) The variation of the flux ratio vs the rigidity for different L1 entry regions (see inset). c) (right) and d) The variation of the flux ratio measured using only the inner tracker (L2 to L8) or (L1 to L8) vs the full tracker (L1 to L9). (see text for details)

Figures 2(a) show the dependence of the integral of the fluxes above $30 \mathrm{GV}$, i.e., above the maximum geomagnetic cutoff, on the angle $\theta$ between the incoming particle direction and the AMS $z$ axis; this verifies the systematic errors assigned to the acceptance. Figures 2(b) show the monthly integral fluxes above $45 \mathrm{GV}$ are within the systematic errors. This verifies that the detector performance is stable over time and that the fluxes above $45 \mathrm{GV}$ show no observable effect from solar modulation fluctuations for this measurement period. The variation of the proton flux due to solar modulation will be the subject of a separate publication. Figure 2(c, left) shows that the ratios of proton fluxes obtained using events which pass through different sections of L1 to the average flux is in good agreement and within the assigned systematic errors; this verifies the errors assigned to the tracker alignment. 
Figures 2(c, right) and 2(d, left) show that the proton and helium fluxes obtained using the rigidity measured by only the inner tracker are in good agreement with the fluxes measured using the full lever arm. The flux ratios use the two different event samples corresponding to the inner tracker acceptance and to the L1 to L9 acceptance used for the results presented here. This verifies the systematic errors from the acceptance, the unfolding procedure, and the rigidity resolution function for two extreme and important cases. First, at the MDR of the inner tracker, where the unfolding effects and resolution functions of the inner tracker and the full lever arm are very different. Second, at low rigidities (2 to $10 \mathrm{GV}$ ) where the unfolding effects and the tails in the resolution functions of the inner tracker and full lever arm are also very different due to multiple and nuclear scattering. Lastly, Fig. 2(d, right) shows the good agreement between the helium flux obtained using the rigidity measured by tracker L1 to L8, MDR 1.4 TV, and the full lever arm, MDR 3.2 TV, again using different event samples, thus verifying the systematic errors on the rigidity resolution function over this extended rigidity range.

Most importantly, for both protons and helium several independent analyses were performed on the same data sample by different study groups. The results of those analyses are consistent with the ones presented here.

\section{Results}

The measured proton flux can be found in Ref. [11] in Table I and the helium flux is published in Ref. [12] in Table I as a function of the rigidity at the top of the AMS detector. The contributions of individual sources to the systematic errors are added in quadrature to arrive at the total systematic uncertainty of the proton an helium flux. The Monte Carlo event samples have sufficient statistics such that they do not contribute to the errors. Figure 3(a) on the left shows the proton flux as a function of rigidity with the total errors. In this and the subsequent figures, the points are placed along the abscissa at $\tilde{R}$ calculated for a flux $\propto R^{-2.7}$ [33]. Figure 3(b) on the left shows the AMS proton flux as a function of kinetic energy $E_{\mathrm{K}}$ together with the most recent results (i.e., from experiments after the year 2000). Figure 3(a) on the right shows the helium flux as a function of rigidity with the total errors. Figure 3(b) on the right shows the AMS helium flux as a function of kinetic energy per nucleon $E_{K}$ together with the most recent results (i.e., from experiments after the year 2000).

A power law with a constant spectral index $\gamma$

$$
\Phi=C R^{\gamma},
$$

where $R$ is in $\mathrm{GV}$ and $C$ is a normalization factor, does neither fit the proton flux nor the helium flux at the $99.9 \%$ C.L. for $R>45 \mathrm{GV}$ taken solar modulation into account through the force-field approximation $[34,35]$. We therefore fit the fluxes with a modified spectral index [36]

$$
\Phi=C\left(\frac{R}{45 \mathrm{GV}}\right)^{\gamma}\left[1+\left(\frac{R}{R_{0}}\right)^{\Delta \gamma / s}\right]^{s}
$$

where $s$ quantifies the smoothness of the transition of the spectral index from $\gamma$ for rigidities below the characteristic transition rigidity $R_{0}$ to $\gamma+\Delta \gamma$ for rigidities above $R_{0}$. Fitting the flux above $45 \mathrm{GV}$ yields the following results for protons: $\chi^{2} /$ d.f. $=25 / 26$ with $\gamma=-2.849 \pm 0.002(\text { fit })_{-0.003}^{+0.004}(\mathrm{sys})_{-0.003}^{+0.004}(\mathrm{sol})$, $\Delta \gamma=0.133_{-0.021}^{+0.032}(\mathrm{fit})_{-0.030}^{+0.046}(\mathrm{sys}) \pm 0.005$ (sol), $s=0.024_{-0.013}^{+0.020}(\mathrm{fit})_{-0.016}^{+0.027}(\mathrm{sys})_{-0.004}^{+0.006}(\mathrm{sol})$, and $R_{0}=$ $336_{-44}^{+68}(\mathrm{fit})_{-28}^{+66}(\mathrm{sys}) \pm 1(\mathrm{sol}) \mathrm{GV}$; and these are the results for helium: $\chi^{2} /$ d.f. $=25 / 27$ with $\gamma=$ $-2.780 \pm 0.005$ (fit) \pm 0.001 (sys) \pm 0.004 (sol), $\Delta \gamma=0.119_{-0.010}^{+0.013}(\mathrm{fit})_{-0.028}^{+0.033}$ (sys) \pm 0.004 (sol), $s=$ $0.027_{-0.010}^{+0.014}(\mathrm{fit})_{-0.013}^{+0.017}(\mathrm{sys}) \pm 0.002$ (sol), and $R_{0}=245_{-31}^{+35}(\mathrm{fit})_{-30}^{+33}(\mathrm{sys}) \pm 3$ (sol) GV. 

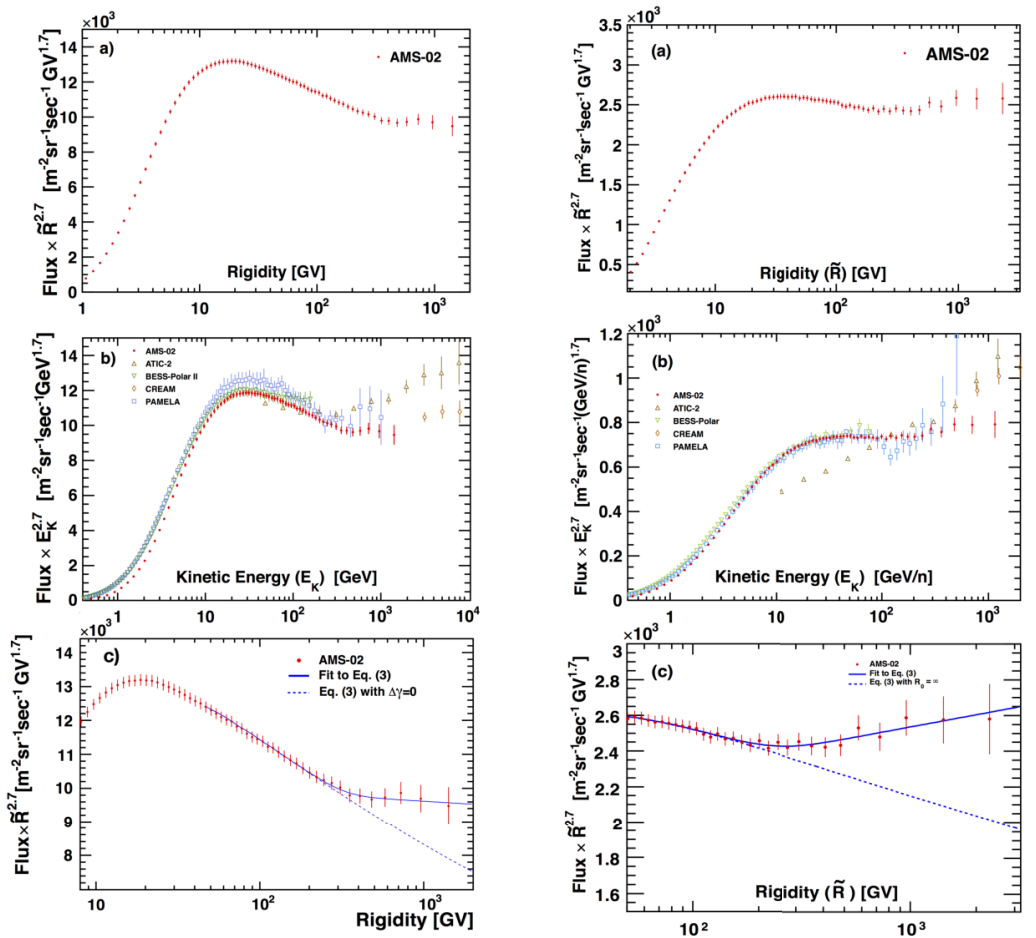

Figure 3: a) The AMS proton and helium flux multiplied by $\tilde{R}^{2.7}$ and total error as a function of rigidity. b) The fluxes as a function of kinetic energy per nucleon $E_{\mathrm{K}}$ as multiplied by $E_{\mathrm{K}}^{2.7}$ compared with recent measurements [3-6, 8, 9]. For the AMS proton results $E_{\mathrm{K}} \equiv \sqrt{\tilde{R}^{2}+M_{p}^{2}}-M_{p}$ where $M_{p}$ is the proton mass. For the AMS helium results $E_{K} \equiv\left(\sqrt{4 \tilde{R}^{2}+M^{2}}-M\right) / 4$ where $M$ is the ${ }^{4} \mathrm{He}$ mass. c) The fluxes as fitted with Eq. (3). For illustration, the fit results with $\Delta \gamma$ set to zero or $R_{0}=\infty$ are also shown.

The first error quoted (fit) takes into account the statistical and uncorrelated systematic errors of the flux. The second (sys) is the error from the remaining systematic errors, namely from the rigidity resolution function and unfolding, and from the absolute rigidity scale, with their bin-to-bin correlations accounted for using the migration matrix $M_{i j}$. The third (sol) is the uncertainty due to the variation of the solar potential $\phi=0.50$ to $0.62 \mathrm{GV}$ [34]. The fits confirm that above $45 \mathrm{GV}$ the fluxes are incompatible with a single spectral index at the $99.9 \%$ C.L. The fits are shown in Fig. 3(c) on the left for protons and 3(c) on the right for helium. For illustration, the fit results with $\Delta \gamma$ set to zero or $R_{0}=\infty$ are also shown.

To obtain the detailed variation of $\gamma$ with rigidity for the proton and helium flux in a model independent way, the spectral index is calculated from

$$
\gamma=d[\log (\Phi)] / d[\log (R)]
$$

over independent rigidity intervals above $8.48 \mathrm{GV}$, with a variable width to have sufficient sensitivity to determine $\gamma$. The results are presented in Figure 4(a). 
As seen, the magnitude of the helium spectral index is different from that of the proton spectral index but the rigidity dependence is similar for helium and protons. In particular, the spectral indices both progressively harden with rigidity above $\sim 100 \mathrm{GV}$.

To examine the difference between the rigidity dependence of the proton and helium fluxes, the ratio of the proton flux to the helium flux, or $\mathrm{p} / \mathrm{He}$ ratio, was computed in the range where they overlap, from $1.9 \mathrm{GV}$ to $1.8 \mathrm{TV}$. The statistical errors are the sum in quadrature of the relative statistical errors of the proton and helium fluxes multiplied by the $\mathrm{p} / \mathrm{He}$ ratio. The systematic errors from the trigger and acceptance are likewise added in quadrature. The correlations in the systematic errors from the unfolding and the absolute rigidity scale between the proton and helium fluxes have been accounted in calculating the corresponding systematic errors of the $\mathrm{p} / \mathrm{He}$ ratio. The contribution of individual sources to the systematic error are added in quadrature to arrive at the total systematic uncertainty. Figure 4(b) shows the AMS measurement with total errors, the quadratic sum of statistical and systematic errors, together with other recent measurements.

Above $45 \mathrm{GV}$ the $\mathrm{p} / \mathrm{He}$ ratio measured by AMS is well fit with a single power law, Eq. (2), with a $\chi^{2} / d . f .=22 / 29$ and a spectral index of $\gamma_{\mathrm{p} / \mathrm{He}}=-0.077 \pm 0.002$ (fit) \pm 0.007(sys). The first error quoted (fit) takes into account the statistical and uncorrelated systematic errors from the flux ratio reported in [12], Table II. The second (sys) is the error from the remaining systematic errors, namely from the rigidity resolution function, unfolding and proton and helium inelastic cross sections, with proper accounting of the bin-to-bin correlations.
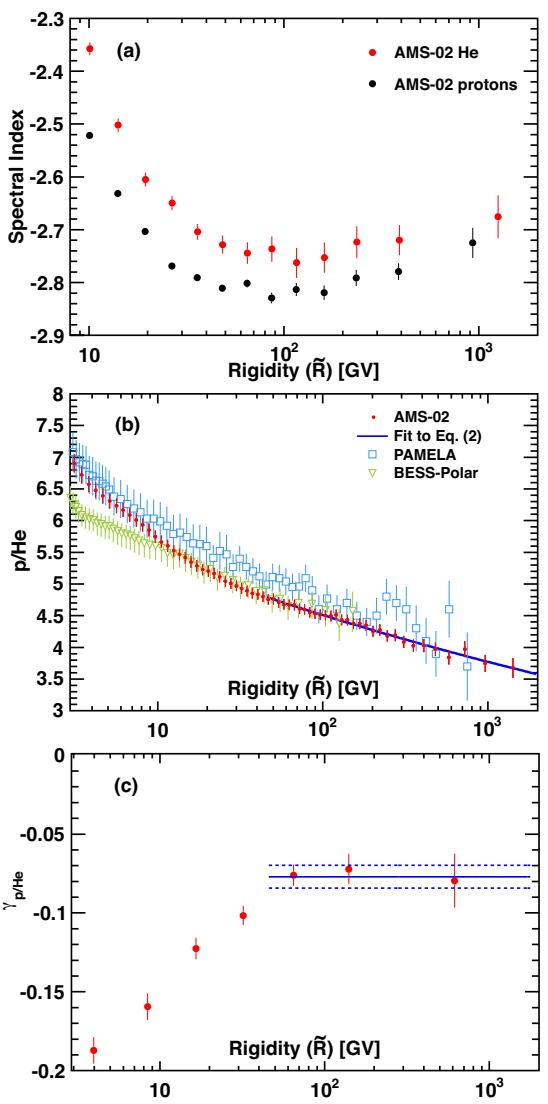

Figure 4: (a) The dependence of the helium and proton spectral indices on rigidity. (b) The $\mathrm{p} / \mathrm{He}$ ratio as a function of rigidity compared with recent measurements $[4,6]$. The solid blue curve indicates the fit of a single power law, Eq. (2), to the AMS data. (c) The rigidity dependence of the $\mathrm{p} / \mathrm{He}$ spectral index as measured by AMS. Above $45 \mathrm{GV}$ it becomes constant at $\gamma_{\mathrm{p} / \mathrm{He}}=-0.077 \pm 0.002$ (fit) \pm 0.007 (sys) as indicated by the blue lines.

Figure 4(c) shows the detailed variation of $\gamma_{\mathrm{p} / \mathrm{He}}$ with rigidity over non-overlapping rigidity intervals. As seen, the spectral index of the ratio increases with rigidity up to about $45 \mathrm{GV}$. Above $45 \mathrm{GV}$ it becomes constant. 


\section{Conclusion}

In conclusion, precise knowledge of the proton and helium flux is important in understanding the origin, acceleration, and propagation of cosmic rays. Our precise measurements of the proton and helium flux show that the fluxes deviate from a single power law and the spectral indices progressively harden at rigidities larger than $100 \mathrm{GV}$. The magnitude of the helium spectral index is different from that of the proton spectral index, but the rigidity dependence is similar for helium and protons. The proton to helium flux ratio spectral index increases with rigidity up to about $45 \mathrm{GV}$ and becomes constant for higher rigidities, i.e., the proton to helium flux ratio is well described by a single power law above $45 \mathrm{GV}$.

\section{References}

[1] I. A. Grenier, J. H. Black and A. W. Strong, Annu. Rev. Astron. Astrophys. 53, 199 (2015); P. Blasi, Astron. Astrophys. Rev. 21, 70 (2013); A. W. Strong, I. V. Moskalenko, and V. S. Ptuskin, Annu. Rev. Nucl. Part. Sci. 57, 285 (2007).

[2] M. Hareyama et al., J. Phys. Conf. 31, 159 (2006); M. Boezio et al., Astropart. Phys. 19, 583 (2003); E. Diehl, D. Ellithorpe, D. Muller, S.P. Swordy, Astropart. Phys. 18, 487 (2003); J. Alcaraz et al., Phys. Lett. B 490, 27 (2000); W. Menn et al., Astrophys. J. 533, 281 (2000); R. Bellotti et al., Phys. Rev. D 60, 052002 (1999); M. Boezio et al., Astrophys. J. 518, 457 (1999); K. Asakimori et al., Astrophys. J. 502, 278 (1998); M. Ichimura et al., Phys. Rev. D 48, 1949 (1993); I. P. Ivanenko et al., in Proceedings of the 23rd International Cosmic Ray Conference, Calgary, 17 (1993).

[3] For the ATIC experiment see A. D. Panov et al., Bull. Russian Acad. Sci. 73, 564 (2009) extracted from D. Maurin, F. Melot, R. Taillet, Astron. Astrophys. 569, A32 (2014).

[4] We are grateful to the BESS-Polar Collaboration for providing the BESS-Polar II proton spectrum, measured during a 25-day Antarctic balloon flight in December 2007 and January 2008. Statistical and systematic errors are combined. A publication detailing these measurements is in preparation. For a description of the BESS-Polar experiment see K. Abe et al., Phys. Rev. Lett. 108, 051102 (2012). For the BESS experiment see Y. Shikaze et al., Astropart. Phys. 28, 154 (2007); S. Haino et al., Phys. Lett. B 594, 35 (2004); T. Sanuki et al., Astrophys. J. 545, 1135 (2000).

[5] For the CREAM experiment see Y. S. Yoon et al., Astrophys. J. 728, 122 (2011).

[6] For the PAMELA experiment see O. Adriani, et al., Astrophys. J. 765, 91 (2013); O. Adriani, et al., Science 332, 69 (2011).

[7] V. A. Derbina et al., Astrophys. J. 628, L41 (2005); M. Boezio et al., Astropart. Phys. 19, 583 (2003); E. Diehl, D. Ellithorpe, D. Muller, and S.P. Swordy, Astropart. Phys. 18, 487 (2003); J. Alcaraz et al., Phys. Lett. B 494, 193 (2000); W. Menn et al., Astrophys. J. 533, 281 (2000); R. Bellotti et al., Phys. Rev. D 60, 052002 (1999); M. Boezio et al., Astrophys. J. 518, 457 (1999); K. Asakimori et al., Astrophys. J. 502, 278 (1998); I. P. Ivanenko et al., in Proceedings of the 23rd International Cosmic Ray Conference, Calgary (World Scientific, Singapore, 1993) p. 17.

[8] For the ATIC experiment see A. D. Panov et al., Bull. Russian Acad. Sci. 73, 564 (2009) extracted from D. Maurin, F. Melot, and R. Taillet, Astron. Astrophys. 569, A32 (2014).

[9] For the CREAM experiment see Y. S. Yoon et al., Astrophys. J. 728, 122 (2011).

[10] See, for example, A. D. Erlykin and A. W. Wolfendale, J. Phys. G: Nucl. Part. Phys. 42, 075201 (2015); S. Thoudam and J. R. Hörandel, Astronom. Astrophys. 567, A33 (2014); G. Bernard, T. Delahaye, Y.-Y. Keum, W. Liu, P. Salati, and R. Taillet, Astronom. Astrophys. 555, A48 (2013); V. S. Ptuskin, V. Zirakashvili, and E. S. Seo, Astrophys. J. 763, 47 (2013); N. Tomassetti, Astrophys. J. Lett. 752, L13 (2012); P. Blasi, E. Amato, and P. D. Serpico, Phys. Rev. Lett. 109, 061101 
(2012); A. E. Vladimirov, G. Jóhannesson, I. V. Moskalenko, and T. A. Porter, Astrophys. J. 752, 68 (2012); M. A. Malkov, P. H. Diamond, and R. Z. Sagdeev, Phys. Rev. Lett. 108, 081104 (2012); L. A. Fisk and G. Gloeckler, Astrophys. J. 744, 127 (2012); Y. Ohira and K. Ioka, Astrophys. J. Lett. 729, L13 (2011).

[11] M. Aguilar et al., Phys. Rev. Lett. 114, 171103 (2015);

[12] M. Aguilar et al., Phys. Rev. Lett. 115, 211101 (2015);

[13] A. Kounine, Int. J. Mod. Phys. E 211230005 (2012); S. Rosier-Lees, in Proceedings of Astroparticle Physics TEVPA/IDM, Amsterdam, 2014 (to be published); S. C. C. Ting, Nucl. Phys. B, Proc. Suppl. 243-244, 12 (2013); S.-C. Lee, in Proceedings of the 20th International Conference on Supersymmetry and Unification of Fundamental Interactions (SUSY 2012), Beijing, 2012 (unpublished); M. Aguilar, in Proceedings of the XL International Meeting on Fundamental Physics, Centro de Ciencias de Benasque Pedro Pascual, 2012 (unpublished); S. Schael, in Proceedings of the 10th Symposium on Sources and Detection of Dark Matter and Dark Energy in the Universe, Los Angeles, 2012 (unpublished); B. Bertucci, Proc. Sci. EPS-HEP, 67 (2011); M. Incagli, AIP Conf. Proc. 1223, 43 (2010); R. Battiston, Nucl. Instrum. Methods Phys. Res., Sect. A 588, 227 (2008).

[14] K. Luebelsmeyer et al., Nucl. Instrum. Methods Phys. Res., Sect. A 654, 639 (2011).

[15] B. Alpat et al., Nucl. Instrum. Methods Phys. Res., Sect. A 613, 207 (2010).

[16] V. Bindi et al., Nucl. Instrum. Methods Phys., Sect. A 743, 22 (2014) and references therein.

[17] J. Allison et al., IEEE Trans. Nucl. Sci. 53, 270 (2006); S. Agostinelli et al., Nucl. Instrum. Methods Phys. Res., Sect. A 506, 250 (2003).

[18] A. Boudard, J. Cugnon, J.-C. David, S. Leray, and D. Mancusi Phys. Rev. C 87014606 (2013); S. Leray, D. Mancusi, P. Kaitaniemi, J. C. David, A. Boudard, B. Braunn, and J. Cugnon J. Phys.: Conf. Series 420, 012065 (2013).

[19] J. Ranft, Phys. Rev. D 51, 64 (1995).

[20] V. G. Ableev et al., Acta Physica Polonica B 16, 913 (1985).

[21] J. Alcaraz et al., Phys. Lett. B 484, 10 (2000); see also Ref. [24].

[22] C. C. Finlay et al. Geophys. J. Int. 183/3, 1216 (2010).

[23] E. Vannuccini, in Proceedings of the 28th International Cosmic Ray Conference, Tsukuba, 1801 (2003).

[24] M. Aguilar et al., Physics Reports 366/6, 331 (2002).

[25] The AMS Collaboration, Measurement of the Flux of Light Nuclei in Primary Cosmic Rays with the Alpha Magnetic Spectrometer on the International Space Station, (to be published).

[26] M. Aguilar et al., Phys. Rev. Lett. 113, 121102 (2014).

[27] N. Abgrall et al. Phys. Rev. C 84, 034604 (2011); J. R. Letaw, R. Silberberg, C. H. Tsao, Astrophys. J. Supp. 51271 (1983); A. S. Carroll et al., Phys. Lett. B 80, 319 (1979); S. P. Denisov, S. V. Donskov, Yu. P. Gorin, R. N. Krasnokutsky, A. I. Petrukhin, Yu. D. Prokoshkin, D. A. Stoyanova, Nucl. Phys. B 61, 62 (1973); G. Bellettini, G. Cocconi, A. N. Diddens, E. Lillethun, G. Matthiae, J. P. Scanlon, A. M. Wetherell, Nucl. Phys. 79, 609 (1966); T. Bowen, M. Di Corato, W. H. Moore, G. Tagliaferri, Il Nuovo Cimento 9, 908 (1958).

[28] A. Ingemersson et al., Nucl. Phys. A 676, 3 (2000); A. Auce, R. F. Carlson, A. J. Cox, A. Ingemarsson, R. Johansson, P. U. Renberg, O. Sundberg, G. Tibell, and R. Zorro, Phys. Rev. C 50, 871 (1994); I. Tanihata et al., Phys. Lett. B 160, 380 (1985); V. D. Aksinenko et al., Nucl. Phys. A 348, 518 (1980); E. O. Abdrahmanov et al., Z. Phys. C 5, 1 (1980); J. Jaros et al., Phys. Rev. C 18, 2273 (1978). 
[29] The AMS Collaboration, Measurement of Nuclear Interaction Cross Sections with the Alpha Magnetic Spectrometer on the International Space Station, (to be published).

[30] The AMS Collaboration, Measurement of the helium isotopic composition in Primary Cosmic Rays with the Alpha Magnetic Spectrometer on the International Space Station, (to be published); O. Adriani et al., Astrophys. J. 770, 2 (2013); B. Coste, L. Derome, D. Maurin, and A. Putze, Astronom. Astrophys. 539, A88 (2012); M. Aguilar et al., Astrophys. J. 736, 105 (2011); Z. D. Myers et al., in Proceedings of the 28th International Cosmic Ray Conference, Tsukuba, p. 1805 ; J. Z. Wang et al., Astrophys. J. 564, 244 (2002); S. P. Ahlen et al., Astrophys. J. 534, 757 (2000); O. Reimer et al., Astrophys. J. 496, 490 (1998); Y. Hatano, Y. Fukada, T. Saito, H. Oda, and T. Yanagita, Phys. Rev. D 52, 6219 (1995); J. J. Beatty et al., Astrophys. J. 413, 268 (1993); S. P. Jordan et al., Astrophys. J. 291, 207 (1985).

[31] F. Giovacchini, Nucl. Instrum. Methods Phys. Res., Sect. A 766, 57 (2014).

[32] L. Accardo et al., Phys. Rev. Lett. 113, 121101 (2014); M. Aguilar et al., Phys. Rev. Lett. 110, 141102 (2013).

[33] G. D. Lafferty, T.R. Wyatt, Nucl. Instr. Methods Phys. Res., Sect. A 355, 541 (1995). We have used Eq. (6) with $\tilde{R} \equiv x_{l w}$.

[34] I. G. Usoskin, G. A. Bazilevskaya, G. A. Kovaltsov, J. Geophys. Res. 116 A02104 (2011); K. G. McCracken, J. Beer, J. Geophys. Res. 112 A10101 (2007); I. G. Usoskin, K. Alanko-Huotari, G.A. Kovaltsov,K. Mursula, J. Geophys. Res. 110 A12108 (2005); We have also used more recent data from: http://cosmicrays.oulu.fi/phi/Phi_mon.txt.

[35] L. J. Gleeson, W. I. Axford, Astrophys. J. 154, 1101 (1968).

[36] K. Beuermann et al., Astron. Astrophys. 352, L26 (1999). 